Revue d'histoire de l'Amérique française

26. REVUE D.HISTOIRE DE L'AMÉRIQUE FRANÇAISE

\title{
On a vieilli Champlain
}

\section{Jean Liebel}

Volume 32, numéro 2, septembre 1978

URI : https://id.erudit.org/iderudit/303691ar

DOI : https://doi.org/10.7202/303691ar

Aller au sommaire du numéro

Éditeur(s)

Institut d'histoire de l'Amérique française

ISSN

0035-2357 (imprimé)

1492-1383 (numérique)

Découvrir la revue

Citer cet article

Liebel, J. (1978). On a vieilli Champlain. Revue d'histoire de l'Amérique française, 32(2), 229-237. https://doi.org/10.7202/303691ar d'utilisation que vous pouvez consulter en ligne.

https://apropos.erudit.org/fr/usagers/politique-dutilisation/ 


\section{ON A VIEILLI CHAMPLAIN}

JEAN LIEBEL

Bordeaux, France

Depuis plus d'un siècle, les biographes de Samuel Champlain nous font croire qu'il serait né vers ou entre les années 1567 et 1570; de ce fait, ils le vieillissent car, en réalité, il naquit bien après ces années-là. Ils affirment aussi que le lieu de sa naissance est Brouage, en Saintonge.

Certes, il est possible, sinon probable, que Champlain ait vu le jour dans cette ville, mais pour être précis, les textes connus ne le révèlent pas exactement. Faute de pouvoir consulter son acte de baptême, qui est perdu ou n'a jamais été écrit sur un registre, plusieurs textes mentionnent seulement que ce personnage est «de Brouage»; ce sont, notamment, le titre de son livre publié en 1604: Des sauvages ou voyage de Samuel Champlain de Brouage... et les quelques mots, en langue anglaise, qui précèdent la déclaration qu'il fit le 9 novembre 1629, à Londres, par-devant un juge de l'Amirauté: "Samuel Champlain of Browage in Guien in the Kingdom of France gent ${ }^{1}{ }^{1}$ L'expression «de Brouage» indique le lieu habituel de résidence du personnage, mais il peut, à cette époque, signifier aussi le lieu de naissance. Nous en avons pour preuve un écrit de Champlain qui précise que François Gravé, sieur du Pont, était «de Sainct Malo»; or ce François Gravé est bien né dans cette ville, ainsi qu'il sera vu plus loin. Enfin Champlain possédait à Brouage plusieurs maisons, dont l'une lui venait de sa grand-mère maternelle. Pour toutes ces raisons, il est convenable d'admettre que le lieu de naissance de Champlain pourrait être Brouage, mais l'affirmer ne l'est pas.

1 Le document original est conservé à Richemont en Angleterre, au « Public Record Office», sous la cote CO $\mathrm{I} / 5 \mathrm{n}^{\circ} 34$; résumé dans «Public Record Office Calendar Colonial Serie 1574-1600», 102, 103; Robert Le Blant, in Bulletin philosophique et historique (Paris Impr. Nle, 1960): 368, indique ce résumé, mais ne le reproduit pas. 
Les auteurs qui font naître Champlain dans les années 1567 ou 1570 se sont bornés à reproduire ce que d'autres, avant eux, avaient écrit, à l'exception bien entendu des deux premiers qui eurent l'idée d'imaginer, l'un de le faire naitre «en 1567 » et l'autre «vers $1570 »$.

Avant le milieu du XIX ${ }^{\mathrm{e}}$ siècle, aucun écrivain ne s'était intéressé à l'année de naissance de Champlain. Il faut attendre 1851 pour qu'un nommé Pierre Damien Rainguet écrive, de Champlain, dans Biographie saintongeaise ...: «Ce navigateur célèbre naquit à Brouage d'une famille de pêcheurs, en 1567. ${ }^{2}$ L'auteur n'indiquait pas de source et il ajoutait ainsi une erreur aux centaines d'autres qui abondent dans ce livre. De cet écrivain «dont la gaieté de son caractère pétillait et éclatait en saillies spirituelles ${ }^{3}$, on est en droit de se demander s'il n'était pas quelque peu farceur, notamment quand il raconte certaines histoires aux allures de canulars ${ }^{4}$. Des personnes s'en sont émues, notamment Léopold Delayant, qui, en 1867, juge que l'année 1567, donnée par Rainguet pour être celle de la naissance de Champlain, résulte d'une "conjoncture " 5 .

Peu après la publication de cette biographie fantaisiste de Champlain, un artiste reproduit le portrait, datant de 1654, d'un contrôleur général des finances et a l'audace d'écrire en dessous que c'est celui de Champlain, en précisant, comme Rainguet: "né à Brouage en 1567 ». Lithographié, imprimé, puis importé au Canada en 1854, ce portrait, plus ou moins modifié dans les détails, illustrera de nombreuses publications. Il dupera aussi plusieurs auteurs, entre autres, Pierre Margry, en $1864^{6}$, jusqu'à ce que la supercherie soit enfin dé-

2 Pierre Damien Rainguet, Biographie saintongeaise ou dictionnaire historique de tous les personnages qui... (Saintes, chez M. Niox, 1851).

$3 \mathrm{H}$. Feuilleret et L. de Richemond, Biographie de la Charente-inférieure Niort La Rochelle (1875), 633, qui précisent aussi que cet auteur était notaire à SaintFort-sur-Gironde, où il mourut le 23 mars 1875, âgé de 72 ans. Une lettre écrite et signée de lui révèle son caractère (Bibl. Mun. La Rochelle ms338 $\mathrm{f}^{\circ} 180$ ).

4 Certaines sont mises en notes par M. Faye, d'après l'exemplaire de la Bibliothèque municipale de Saintes; d'autres, une trentaine, dans le Bulletin des archives historiques de la Saintonge... 1879-1893 ; mais quantité d'autres n'ont pas été publiées. L'une des plus remarquables est celle de faire passer Anthoine, seigneur d'Authon, pour avoir été un pirate barbaresque! III, IV : 1

5 Léopol Delayant, Notice sur Samuel Champlain... (Niort L. Clouzot, 1867),

6 Sans nom d'auteur, "Les pionniers et la nationalité française au Canada», in Recueil des actes de la commission des arts et monuments historiques de la Charente Inférieure, I (1864): 457. 
couverte en $1904^{7}$. Malgré cette révélation, les portraits proposés et vendus comme étant celui de Champlain continueront, jusqu'à nos jours, à avoir un succès considérable. Tous reproduits d'après un portrait qui n'est pas celui de Champlain - un fait est certain - la mention «né à Brouage en 1567» n'est pas une preuve, elle a été vraisemblablement tirée du livre de Rainguet.

En 1870, dans la préface de ce qu'il titre Oeuvres de Champlain, l'abbé Laverdière donne une biographie du personnage; malheureusement trompé par un texte mal lu et un autre mal interprété, il manque de peu l'occasion de déceler l'erreur de Rainguet. Tout au contraire, il la confirme et lui donne un essor prodigieux ${ }^{8}$.

C'est en 1878 qu'apparaît, pour la première fois, gravée dans la pierre d'un monument, à Brouage, l'inscription indiquant que Champlain serait né «vers $1570 »$; elle est due vraisemblablement au conseiller général de la Charente inférieure, qui incita l'Assemblée départementale à en financer la construction: Omer Charlet ${ }^{9}$. Il ne semble pas que ce peintre de tableaux à sujets religieux destinés à orner les églises ${ }^{10}$, ait laissé le moindre écrit pouvant révéler d'où il tira la preuve d'une naissance de Champlain «vers $1570 »$. Peutêtre avait-il remarqué combien il était anormal, en pleine guerre de religion, de faire naître ce personnage, considéré pour avoir été catholique, dans une ville occupée par les huguenots. Le fait que les catholiques étaient maîtres de la ville une partie de l'année 1570 lui aurait donné l'idée de le faire naître cette année-là.

Après l'apparition de cette inscription indiquant que Champlain est né «vers 1570 », on ne sait plus que penser; les uns estiment que Rainguet a raison, d'autres que l'année 1570 est la bonne, mais la plupart déplorent que l'une et l'autre de ces deux années aient été lancées, sans indication de sources ${ }^{11}$. Cette absence de preuve ne

7 Victor Hugo Palsists, "A critical examination of Champlain's portraits», in Acadiensis (1904), IV: 306-311; reproduit partiellement in Bulletin des recherches historiques, 38 (1932): 755-759; complété par H.P. Biggar, «The portrait of Champlain», dans Canadian Historical Review, I (1920): 379-380.

8 Euvres de Champlain, publiées par l'abbé Laverdière; seconde édition (Québec, Imprimerie du Séminaire, 1870), I: ix-x.

9 Archives de la Charente-Maritime, délibérations des 22 déc. 1877 et 20 août 1878.

10 Bulletin des archives historiques de la Saintonge, I (1879): 198; IV (1882): 269; XIII (1893): 271, 272; XIV (1894): 322.

11 Louis Audiat, "Brouage et Champlain, 1578, 1667», documents inédits, dans Archives historiques de la Saintonge, VI (1879): 378; extrait (Paris, Maisonneu- 
gêna nullement les membres du comité chargé de décider du choix de l'inscription à placer sur le monument Champlain à Québec. Entre les deux années, ils choisiront, on ne sait pourquoi, la plus invraisemblable, celle de 1567, au cours de laquelle les huguenots sont maîtres de Brouage ! ${ }^{12}$

La raison pour laquelle ces deux années 1567 et 1570 furent admises assez facilement pour être, l'une ou l'autre, celle de la naissance de Champlain, réside vraisemblablement dans le fait que Champlain aurait été suffisamment âgé pour avoir avant l'année 1598 , les grades, titres ou qualités, que certains imaginèrent de lui attribuer après sa mort, et tout d'abord le grade de maréchal-des-logis ${ }^{13}$. Mais l'indication selon laquelle Champlain aurait été maréchal-deslogis est tirée du trop fameux Brief discours, dont l'origine douteuse, la publication bien après la mort de Champlain, les erreurs et invraisemblances nombreuses font penser qu'il n'a sûrement pas été écrit par Champlain ${ }^{14}$. Certes, quelques erreurs bien compréhensibles se trouvent dans les livres considérés pour avoir été, sans nul doute, écrits par Champlain; elles sont rares et de peu d'importance. Il ne serait pas permis d'égarer le lecteur en des mensonges aussi grossiers que ceux de Brief discours; c'était un honnête homme.

D'autres grades, ou titres, qui lui furent à tort attribués ont, eux aussi, fait penser que Champlain était un homme suffisamment âgé pour assumer ces hautes fonctions: "gouverneur», «lieutenantgénéral pour le roi en Nouvelle France», "gouverneur général de la Nouvelle France», «lieutenant-gouverneur» et quelques autres

ve, 1879), 26; Louis Audiat, "Samuel de Champlain», in Bulletin des archives historiques de la Saintonge, XIII (1893): 246, 248; N.-E. Dionne, Samuel de Champlain, fondateur de Québec... (Québec, A. Côté, 1891), I : 4.

12 Abbé H.R. Casgrain, Notes relatives aux inscriptions du monument de Champlain (Québec, Dussault et Proulx, 1898); sans nom d'auteur, Inauguration du monument Champlain à Québec, le 21 septembre 1898 (Québec, impr. "Le Soleil», 1902).

13 «Brief discours des choses plus remarquables que Samuel Champlain de Brouage a reconnues aux Indes Occidentalles, in Oeuvres de Champlain, op. cit., 1 ; précédemment publiées en anglais par la Hakluyt Society en 1859. Le maréchal des logis était un sous-officier chargé de fournir du fourrage aux chevaux ou le logement aux hommes du régiment ; voir du père G. Daniel, Histoire de la milice française, 2 vol. (Paris, 1721), II : 67-69.

14 L.A. Vigneras, «Les voyages de Samuel Champlain aux Indes occidentales», «Encore le capitaine Provençal», Marcel Delafosse, "L'oncle de Champlain», in Revue d'histoire de l'Amérique française, XI, 2 (sept. 1957): 163-200, XII, 2 (sept. 1958): 208-216, XIII, I (juin 1959) : 545-549. 
inventés à la fantaisie de chacun. À dire vrai, il n'a eu droit à aucun, pour la bonne raison que le roi de France ne les lui accorda pas. Il ne fut, à partir de 1608, que le lieutenant des lieutenants généraux pour le roi en Nouvelle-France, qui se succédèrent les uns après les autres, ainsi qu'en font foi les documents de l'époque.

Enfin le mot «fondateur», que l'on voit, de nos jours, si souvent suivre son nom, évoque un homme non seulement riche et puissant, mais aussi âgé. Tout comme les précédents grades ou titres, il n'en fut pas qualifié de son vivant ${ }^{15}$, parce que, outre le fait que ce titre implique des exigences de fortune que Champlain était loin de posséder, le responsable de l'entreprise qui aboutit à la fondation de Québec n'est pas lui, mais Pierre Dugua, sieur de Mons, lieutenant général du roi en ce qui sera la Nouvelle-France. Associé en 1608 à deux marchands de Rouen, il fournit à Champlain, son lieutenant, les moyens en hommes, matériel et ravitaillement pour lui construire une «habitation», sur les bords de la «rivière de Canada». Prétendre que Champlain est, lui seul, fondateur de Québec est inexact et injuste à l'égard de Pierre Dugua, sieur de Mons.

Ainsi donc, les grades, titres ou qualités dont on a gratifié Champlain après sa mort étant tous abusifs, il n'y a aucune raison de lui supposer un âge en rapport avec eux.

L'étude de plusieurs textes anciens, se complétant les uns les autres, permet non seulement de douter de sa naissance dans les années 1567 ou 1570, mais aussi de fixer, avec assez de précision, vers quelle année il vit le jour. Trois de ceux-ci sont de Champlain lui-même ${ }^{16}$. En 1632, il déclare qu'il a "passé trente huit ans de mon âge à faire plusieurs voyages en mer ${ }^{17}$, ce qui fixe à l'année 1594 ses débuts dans le «métier de la mer». Dans l'éventualité d'une naissance en 1567 ou 1570 , il aurait eu 24 ou 27 ans, âge tout à fait invraisemblable pour commencer à naviguer, même de nos jours.

Les deux autres textes de Champlain concernent François Gravé, sieur du Pont, capitaine de navire, qu'il nomme «le Sieur du

15 Seul, le père Biard écrit, en 1616, qu'il «fonda Québec», mais vraisemblablement, dans le sens de construire (Pierre Biard «Relation de la Nouvelle France» in Relations des Jésuites (Québec, A. Côté, 1858), I : 25.

16 Samuel Champlain, Les voyages de la Nouvelle France occidentale, dicte Canada, faits par le Sr de Champlain... (Paris, Le-Mur, 1632).

17 Samuel Champlain, Traité de la marine et du devoir d'un bon marinier, 2, lequel traité fait suite aux «Voyages de la Nouvelle France occidentale... et a été publié en même temps. 
Pont» ou «Pont Gravé». Dans l'un, il écrit que ce personnage est «de Sainct Malo» ${ }^{18}$, c'est-à-dire, selon toute vraisemblance, né à Saint-Malo; l'autre donne une idée de la différence d'âge entre François Gravé et lui: "Que pour le Sieur du Pont j'estois son amy, \& que son âge me le feroit respecter comme mon père ${ }^{19}$, ce qui indique clairement que François Gravé avait au moins 20 ans de plus que lui, âge à partir duquel, dans la plupart des cas, un homme devient père. Or un texte inédit, confirmé par un autre qui ne l'est pas, permet de connaître l'année de la naissance de ce François Gravé.

Le texte inédit est dans l'un des plus vieux registres de paroisse de France, celui des baptêmes de l'église cathédrale de SaintMalo, en Bretagne. En haut de la page est mentionnée deux fois l'année: «1560»; en face des mots: «françois grave», écrits dans la marge, se lit: "Le mercredy vingtseptiesme jour de novembre an que dessus fut baptize ung filz a Robin grave et guyonne artur sa feme nome françoys par bernard boulain le jeune parrain et jehan michelot comere françoyse poree... ${ }^{20}$. L'acte ne révèle pas la date de naissance, mais il est à présumer qu'elle fut très peu antérieure à celle du baptême, selon la coutume du temps.

Ce François Gravé est très vraisemblablement celui qui, par la suite, sera sieur du Pont, capitaine de navire et ami de Champlain, parce que les autres François Gravé baptisés en cette même unique paroisse de Sainct-Malo ne peuvent nullement être identifiés à lui ${ }^{21}$ et, surtout, à cause du témoignage d'un père récollet qui l'a parfaitement bien connu. En 1629, le père Gabriel Sagard estime «le sieur Pont Gravé vieillard d'aage de plus de 70 ans ${ }^{22}$, fixant ainsi sa naissance en une année plus ou moins antérieure à 1559 , soit à un an près

\footnotetext{
18 Op. cit., 34.

19 Op. cit., 224.
}

20 Arch. d'Ille-et-Vilaine, GG 13, non folioté; copie, pas exactement conforme, GG 8; Robert Le Blant (Bull. phil. et hist... (Paris Impr. Nle, 1960): 369, indique la date de ce baptême, le service où l'acte peut être consulté, mais ne le reproduit pas, ni sa copie. Le parrain Bernard Boulain, le jeune et la «comère» Françoise Porée pourraient être des familles de Richard Boulain et de Thomas Porée, marchands de Saint-Malo, associés à Pierre Dugua, sieur de Mons (par acte du 15 novembre 1613, Nouveaux documents sur Champlain et son époque (Arch. pub. du Canada, Ottawa, 1967), I: 310.

21 Le 5 mai 1567 et le 12 août 1573 , ne semblent pas correspondre au sieur du Pont, selon l'avis donné par M. le Directeur du Service d'Archives d'Ille-et-Vilaine; l'un devint sieur de la Vilaine; l'autre était trop jeune.

22 Gabriel Sagard-Théodat, Histoire du Canada et voyages que les frères mineurs recollets $y$ ont faicts... (Paris, Tross 1866), IV : 884, 891. 


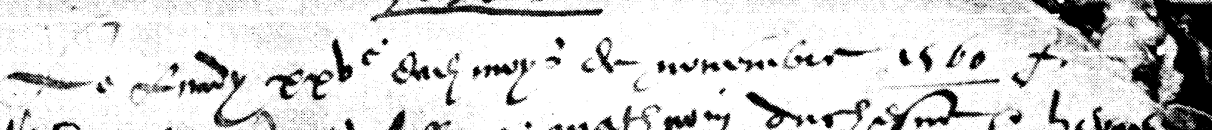

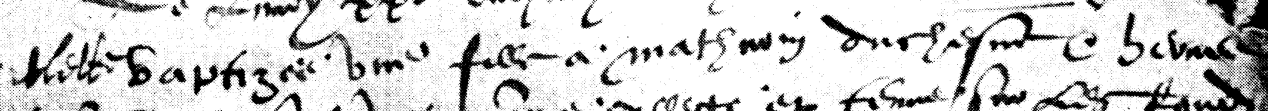

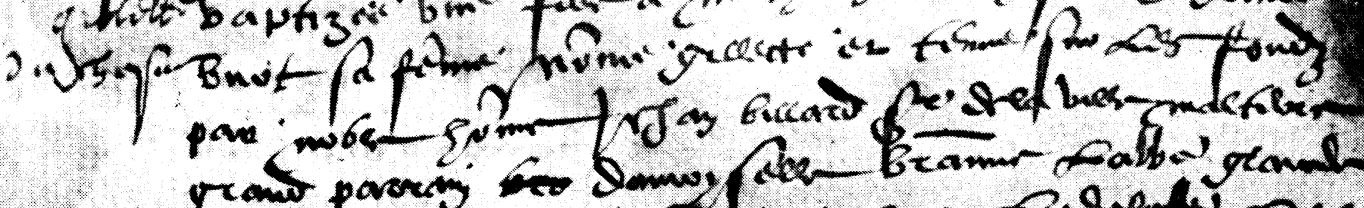

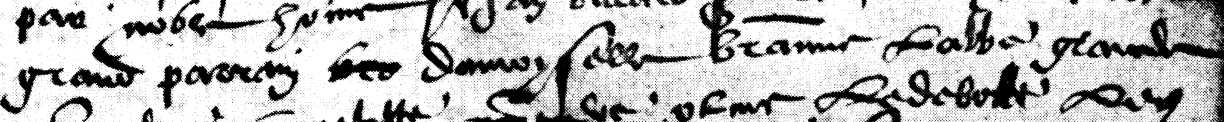

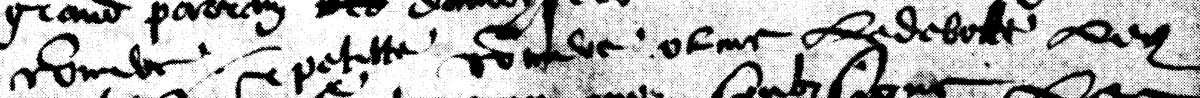

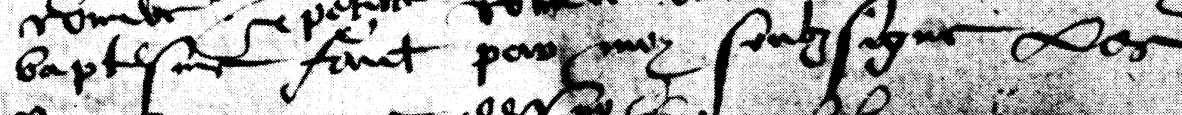

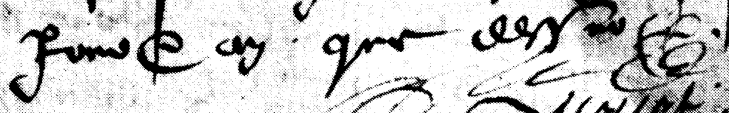

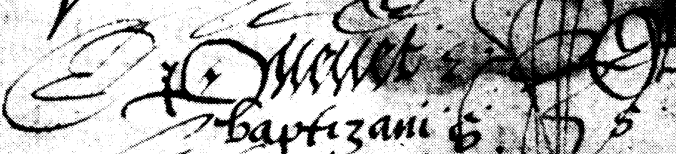

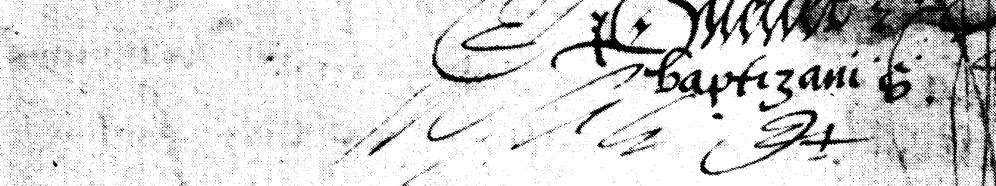

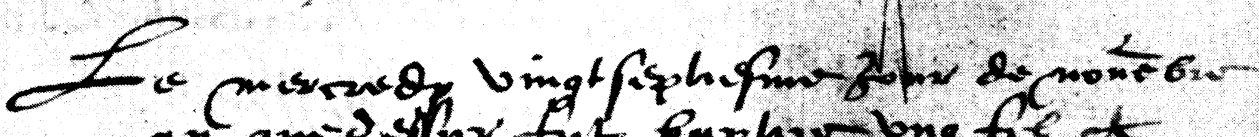

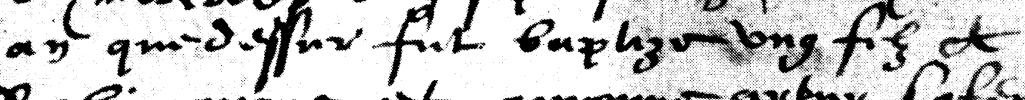

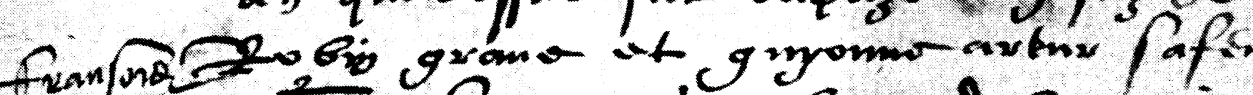

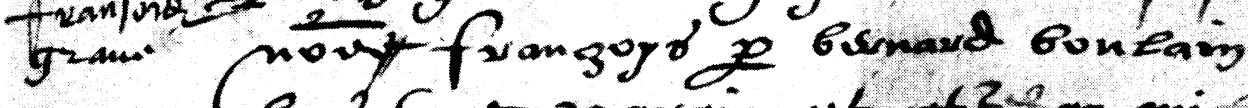

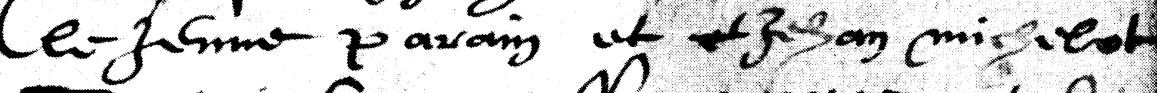

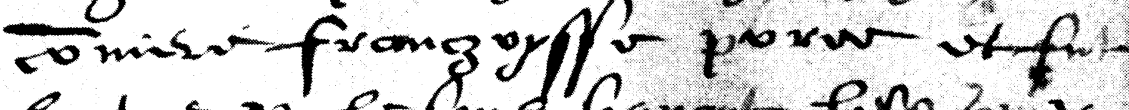

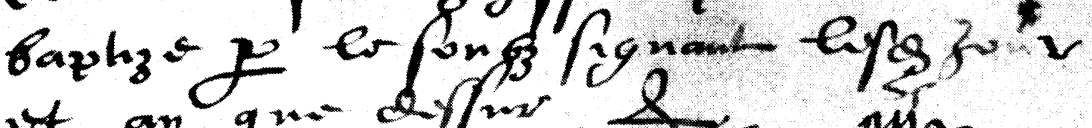
it ay qua dypar
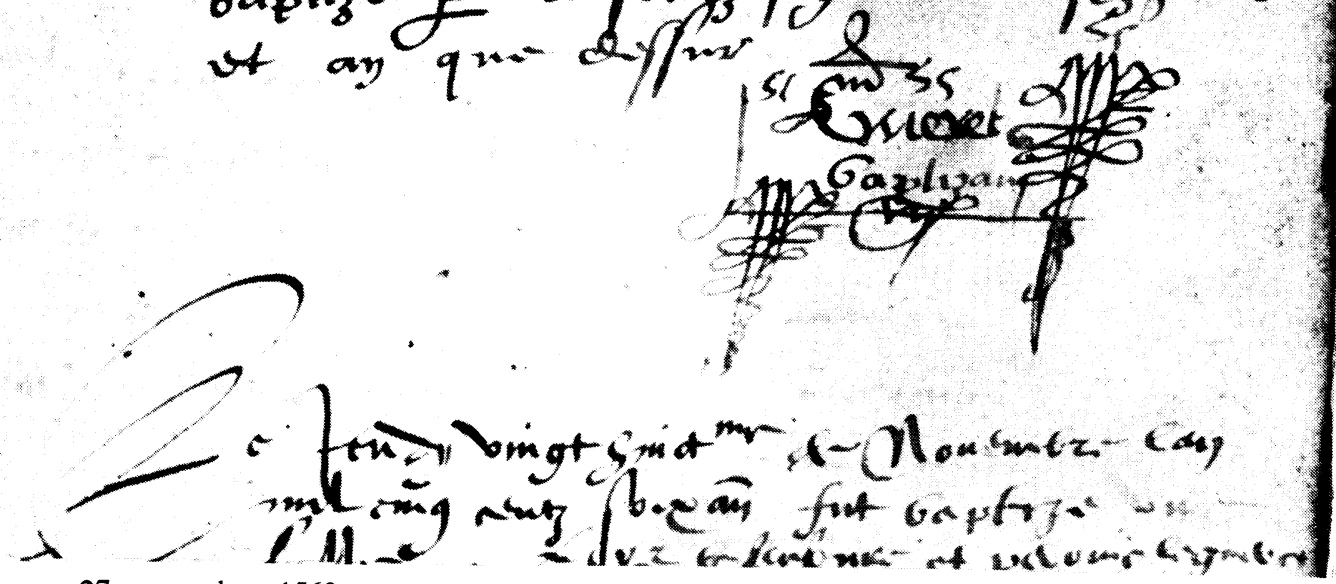

27 novembre 1560 - acte de baptême de François Gravé (le deuxième en partant du haut). Le millésime 1560 est écrit en haut deux fois en chiffres arabes, et en bas en toutes lettres (Arch. d'Ille-et-Vilaine CC 13, photo Arch. Ville de Rennes.) 
celle du baptême en 1560 de ce François Gravé. Erreur d'estimation minime et tout à fait acceptable de la part d'un marin qui a passé sa vie à bourlinguer sur mer. Ainsi donc, François Gravé étant né en 1560 et ayant au moins 20 ans de plus que Champlain, celui-ci serait donc né, au plus tôt, en 1580; d'où l'on peut déduire qu'il ne pouvait avoir que 14 ans en 1594 , ou guère plus.

De l'étude de plusieurs autres textes, il résulte qu'il serait né, au plus tard, en cette même année 1580 , c'est-à-dire qu'il ne pouvait avoir un âge inférieur à 14 ans en 1594 . Évoquant sa jeunesse, Champlain écrit «...pendant les guerres j'avois servi sadite Majesté souz Messeigneurs le Mareschal d'Aumont, de Sainct Luc, \& Mareschal de Brissac. ${ }^{23}$ Or ce Mareschal d'Aumont, commandant les armées du roi avant les deux autres, ayant été tué le 10 août $1595^{24}$, Champlain commença donc à servir avant ce jour, dans le courant de l'année 1595 ou de l'année précédente, mais pas avant. Il ne commença pas à servir avant 1594, à l'âge tout au plus de 14 ans, parce que les garçons d'un âge inférieur sont inutiles aux armées; par contre, il est tout à fait vraisemblable que des garçons de 14 ou 15 ans servent aux armées pendant ces guerres civiles et religieuses qui s'éternisent depuis plus de trente ans. Il ne commença pas à servir avant 1594, parce que pour aller de Brouage en Bretagne, on y va par mer et que nous savons déjà, par un de ses textes, qu'il commença à naviguer précisément en 1594.

Ainsi donc, Samuel Champlain, serait né vers 1580, à Brouage vraisemblablement, où il fut facile de le baptiser catholiquement, cette place étant aux mains du roi depuis 1577. En 1594, il aurait commencé son "métier de la mer», soit quelque temps avant de partir pour la Bretagne, soit à l'occasion de son voyage en cette province car la voie la plus simple et la plus fréquentée est celle de la mer à cette époque. Le jeune Samuel s'embarqua, bien probablement, sur l'un des navires qui, partant de l'important arsenal, allaient ravitaliser les armées du roi en personnel, armes, munitions et victuailles; puis il servit aux armées, non pas forcément au contact de l'ennemi mais plutôt, en considération de son jeune âge, dans quelque service de l'arrière. La paix signée en 1598, alors qu'il est âgé de 18 ans,

${ }^{23}$ Op. cit., 39. Ce «Sainct Luc» est François d'Espinay, gouverneur de Brouage (Jules Sottas, in Bull. arch. hist. Saintonge, XXXIII (1913), XXXIV (1914), XXXVII (1917), XXXVIII (1918).

24 Dom Charles Taillandier, Histoire ecclésiastique et civile de Bretagne (Paris, Delagnette, 1756), II : 447. 
il va, ainsi qu'il l'écrit lui-même, aux «Indes Occidentales », c'est-àdire en Amérique centrale, et il y reste "prés de deux ans» ${ }^{25}$. Au mois de juillet 1601, il a donc environ 21 ans, il est à Cadix, en Espagne, et reçoit d'un nommé Guillaume Hellaine, donation de deux maisons situées à Brouage ${ }^{26}$. En avril 1604, lorsqu'il est invité par Pierre Dugua, sieur de Mons, il s'embarque avec lui sur le Donde-Dieu pour aller en Acadie. Il n'a environ que 24 ans. En 1608, il est encore un jeune homme quand de Mons, ayant remarqué ses qualités, lui confie le soin de lui construire un fort, une «habitation», au lieu nommé Québec; son âge est alors d'environ 28 ans et non pas 38 ou 41 ans, comme l'écrivent certains auteurs, diminuant ainsi ses mérites. Le 27 décembre 1610 , le jour où il signe son contrat de mariage ${ }^{27}$, il n'a guère plus de 30 ans, peut-être ne les a-t-il pas tout à fait. Enfin, ce n'est pas à plus de 65 ans qu'il est mort, le jour de Noël 1635, mais malheureusement, bien plus jeune, tout au plus à l'âge de 55 ans.

25 Op. cit., 39.

26 Mentionnée dans l'acte du 29 déc. 1625, Arch. Nles, Y 165 fo304 v., reproduit dans L'intermédiaire des chercheurs du 30 janv. 1900, 152 et dans Bull. arch. hist. de la Saintonge..., XX (1900): 90.

27 La minute de ce contrat, après avoir été publiée par Emmanuel de Cathelineau (Nova Francia, V, n 3 (mai-juin 1930): 142-155), a été exposée en 1955, malheureusement sous une date et une cote inexactes; $M$. Gonzague de Monts de Savasse a réussi, en 1977, à la retrouver: Arch. Nles, LXXXV: 108; insinuation: Arch. Nles, Y $150 \mathrm{f}^{\circ} 293$. 